\title{
Competencias asociadas a la Atención a la diversidad y la inclusión: perspectiva del profesorado de Educación Infantil
}

Sara María Lao Fernández - Universidad Internacional de la Rioja Roberto Hernández-Soto - Universidad Internacional de la Rioja Dolores Peñalver-García - Universidad Internacional de la Rioja Mónica Gutiérrez-Ortega - Universidad Internacional de la Rioja
(iD) $0000-0002-0910-3327$

(D) $0000-0002-3505-3108$

(i) $0000-0002-9872-1484$

(D) $0000-0002-1536-4240$

Recepción: 09.01.2022 | Aceptado: 14.01.2022

Correspondencia a través de ORCID: Mónica Gutiérrez-Ortega

0000-0002-1536-4240

Citar: Lao Fernández, SM, Hernández-Soto, R, Peñalver-García, D y Gutiérrez-Ortega, M (2022). Atención a la diversidad y Atención Temprana: perspectiva del profesorado de Educación Infantil. REIDOCREA, $11(5), 58-72$.

Área o categoría del conocimiento: Educación

Resumen: La educación inclusiva debe promover la existencia de una variedad de oportunidades de aprendizaje que faciliten la participación del menor en actividades con los adultos y los compañeros y de los apoyos necesarios para que todo ello se produzca. En este proceso, los profesores tienen una función estratégica en la promoción de prácticas que promuevan la atención a la diversidad. Por tanto, las competencias que presenten a nivel didáctico, organizativo, comunicativo e integrativo serán clave para favorecer y no limitar el aprendizaje y la participación en el aula. Del mismo modo, la colaboración con los servicios de Atención Temprana será fundamental para apoyar al alumno de forma integral. El estudio que se presenta se ha llevado a cabo conociendo la percepción de 68 profesores de España a través de la aplicación de un cuestionario adhoc. En los resultados obtenidos destacan que el papel del docente en las escuelas infantiles es fundamental, además de que desarrolle variadas competencias que le ayuden a detectar signos de alarma en el menor, a intervenir adecuadamente a su alumnado de manera personalizada y siendo prioritaria la coordinación con el CDIAT y sus profesionales, para ofrecer más seguridad y empoderamiento al docente en su labor educativa.

Palabra clave: Educación inclusiva

Competencies associated with Attention to Diversity and Inclusion: the perspective of Early Childhood Education teachers

Abstract: Inclusive education should promote the existence of a variety of learning opportunities that facilitate the child's participation in activities with adults and peers and the necessary supports for all of this to occur. In this process, teachers have a strategic role in promoting practices that promote attention to diversity. Therefore, the skills they present at the didactic, organizational, communicative and integrative levels will be key to favor and not limit learning and participation in the classroom. Similarly, collaboration with early intervention services will be essential to support the student in a comprehensive manner. The study presented here has been carried out by knowing the perception of 68 teachers in Spain through the application of an ad hoc questionnaire. The results obtained highlight that the role of the teacher in nursery schools is fundamental, in addition to the development of various competencies that help to detect warning signs in the child, to intervene adequately with their students in a personalized way and to prioritize coordination with the CDIAT and its professionals, to offer more security and empowerment to the teacher in their educational work.

Keyword: Inclusive Education

\section{Introducción}

La División para la Primera Infancia (DEC) y la Asociación Nacional para la Educación de los Niños Pequeños (NAEYC) han señalado que la educación inclusiva en los primeros años de escolarización debe tener en cuenta: (a) el acceso a una variedad de oportunidades de aprendizaje; (b) las adaptaciones individualizadas que facilitan la participación del menor en actividades con los adultos y los compañeros; y (c) los apoyos a nivel de sistema que sustentan los esfuerzos en el aula (DEC y NAEYC, 2009). 
Esta conceptualización apoya la investigación sobre las prácticas inclusivas y las oportunidades de apoyo a los niños en contextos diversos (Love y Horn, 2021).

Por lo tanto, para garantizar el acceso a las oportunidades de aprendizaje es necesario redistribuir las oportunidades de aprendizaje de manera que se contrarresten explícitamente las limitaciones que pueden presentar los menores con alguna discapacidad o reto en su desarrollo.

En este proceso, los profesores van desempeñan un papel clave, sobre todo, a la hora de apoyar la aplicación de políticas y programas educativos. Las investigaciones indican que los profesores son fundamentales para liderar procesos educativos inclusivos y orientados a la atención a la diversidad. Investigaciones previas muestran que los maestros desarrollan funciones estratégicas en la promoción metodologías y prácticas que se ajusten a la edad, a las características del menor y a su cultura, en definitiva, prácticas orientadas a la reducción de las desigualdades en dicha etapa educativa (Fluckiger et al., 2017). Para que la educación infantil sea eficaz, las prácticas docentes deberán atender a la diversidad de los estudiantes ya que así se garantizará el acceso a las actividades y rutinas del aula. Los menores se benefician de los entornos educativos cuando los profesores apliquen prácticas pedagógicas eficaces que estén basadas en la diferenciación, el análisis de tareas y las indicaciones existentes para promover programas inclusivos de calidad (McLeskey et al., 2017). Aspectos que serán posibles si los profesores planifican, enseñan y evalúan teniendo en cuenta la participación y el aprendizaje de todos los alumnos.

Otro aspecto clave para una educación infantil eficaz será la participación y colaboración con las familias, la comunidad y aquellos equipos que apoyan el desarrollo del menor como pueden ser los servicios de Atención Temprana (García et al., 2012). Por lo tanto, su función también será la de unión en el proceso comunicativo entre los diferentes profesionales del centro educativo (orientador, maestro audición y lenguaje, maestro de pedagogía terapéutica, etc.), el profesional de referencia del centro de atención temprana y los cuidadores principales.

Por tanto, para asegurarse de que las prácticas se llevan a cabo en el aula de manera adecuada, el profesorado deberá perseguir un doble objetivo, por un lado, brindar oportunidades de desarrollo a todos los niños independientemente de su condición o capacidades y, por otro, asegurar que se cuentan con métodos de enseñanza adecuados a la variabilidad del alumnado (Grisham-Brown et al., 2005).

\section{Formación del docente sobre las necesidades específicas de apoyo educativo al alumnado}

Para poder tener claros estos objetivos, la Agencia Europea para el Desarrollo de la Educación del Alumnado con Necesidades Educativas Especiales (2012) propone una serie de principios que deberían estar presentes en el perfil profesional del profesorado. Así pues, abogando por una educación inclusiva, describen diferentes valores sobre los cuales es necesario incidir: valorar la diversidad en el aula, apoyar a todo el alumnado, trabajar en equipo y llevar a cabo un desarrollo profesional permanente.

Atendiendo a estos principios y con el propósito de trasladar esta teoría a la práctica en las aulas de educación infantil, existen una serie de intervenciones básicas que van a permitir poder apoyar el aprendizaje y el desarrollo de los alumnos que presenten necesidades educativas especiales. Estas intervenciones giran en torno a conseguir hacer del aula infantil un espacio de calidad, con ajustes del currículo que permitan la participación de todo el alumnado, oportunidades de aprendizaje integradas en las 
actividades cotidianas y estrategias específicas que ayuden a la consecución de logros (Sandall y Schwartz, 2015).

Pero, para que un buen docente actúe correctamente con su alumnado, tenga un rol activo en su actuación educativa, establezca colaboración con las familias (Dunst y Trivette, 2009), demás agentes educativos y de atención temprana, es necesario que tenga unas características innatas, cierta sensibilidad en su trabajo y un don único para la docencia, además de una buena formación académica (Schilling, 2018).

Rodríguez (2019) considera que el docente debe de tener una serie de características propias para ejercer su labor correctamente, sobre todo, en estos primeros años. Algunas de ellas son: empatía, habilidades sociales y de resolución de conflictos, escucha activa, conocimiento de medidas de atención a la diversidad y de los hitos del desarrollo, mirada preventiva ante las dificultades de aprendizaje y desarrollo del niño o niña, fomentar el trabajo en grupo y actitud continua de formación, entre otras.

En la misma línea de lo señalado anteriormente, para que se produzca una eficiente coordinación con otros servicios que prestan apoyo como son los de Atención Temprana también necesaria una adecuada capacitación de todos los profesionales que participan en el proceso de Atención Temprana, incluidos los docentes (Aguaded, et al., 2013).

Si los profesionales están debidamente formados conocerán que servicios y apoyos están a su disposición y sabrán colaborar eficazmente, a través de un trabajo en equipo, para apoyar al alumnado con necesidades educativas especiales en su desarrollo y aprendizaje (Orcajada, 2017)

Ello supone una formación continua que infiere un esfuerzo a lo largo de la vida laboral para mejorar las competencias del docente y para adaptarse a la evidencia científica, de modo que las actuaciones en el aula no sólo vengan marcadas por la formación adquirida en la formación teórica inicial, sino que se adquieran conocimientos también prácticos y de cambio de metodología en una formación permanente (Sandall y Schwartz, 2015). La UNESCO (UNESCO, 2020a, 2020b) señala que, aunque los avances son evidentes todavía hay que hacer mayores esfuerzos en este sentido. En esta línea Cejudo et al, (2016) ya destacado la escasa formación en la atención a la diversidad en los planes de estudios de futuros profesores.

También hay que tener en cuenta que los profesores que tienen un gran abanico de competencias, habilidades, disposiciones y otras experiencias están más dispuestos a promover buenas prácticas de atención a la diversidad (Forlin y Sin, 2017). En la misma línea, algunas investigaciones informan que los profesores pueden limitar las oportunidades de participación de los niños con discapacidad o retos en el desarrollo en los entornos educativos como consecuencia de una formación inadecuada o escasa.

\section{Coordinación de servicios de apoyo a la diversidad}

Es indiscutible decir que para que se pueda dar todo lo indicado es clave que una buena coordinación del docente con la familia y los servicios de apoyo a la diversidad, como son los servicios de atención temprana, ya que ello permite entender mejor al niño o niña con alguna patología o trastorno en el desarrollo y mejorar su intervención diaria en el aula. Pero como demuestra en su estudio Núñez y Duarte (2011), en muchas ocasiones el trasvase de información es poco fluido y dista de la realidad de las prácticas inclusivas y de atención a la diversidad de calidad. Para que ello funcione es necesaria una formación previa, aplicando las recomendaciones recibidas por parte del profesional, escuchando a las familias, ya que son las mayores expertas en sus propios 
hijos e hijas, y llevando a cabo estrategias funcionales para obtener los objetivos planteados entre todos, ante la intervención.

Desde la Atención Temprana se observa cómo las derivaciones de menores con alguna dificultad en su desarrollo, en su mayoría, son iniciadas por el tutor del aula de infantil, ya que por su trato directo con el infante hace que pueda observar algún tipo de señal de alerta (Simón et al., 2021), que hace que salte las alarmas hacia la detección precoz de alguna discapacidad. Pero, la atención a la diversidad del docente no tiene sentido si no está coordinado con los otros agentes implicados en el contexto natural de su alumnado con necesidades educativas especiales (Aranda y de Andrés, 2004).

\section{Objetivos}

El presente estudio persigue un objetivo doble, en primer lugar, explorar cuáles son los niveles reales de competencia que tienen los docentes de Educación Infantil con respecto a la atención a la diversidad. Y, en segundo lugar, analizar la percepción que tiene el profesorado del equipo de profesionales de Centros de desarrollo infantil y atención temprana (CDIAT) y de su relación con los equipos de Atención Temprana.

\section{Método}

En el presente estudio se ha elaborado un instrumento adhoc partiendo de dos cuestionarios previamente validados: el Cuestionario para la Evaluación de Competencias del Profesorado en Atención a la Diversidad (CECPAD) (Mena y García, 2017) y la Escala de Evaluación de la Coordinación del CDIAT con la escuela infantil (García et al., 2012).

Una primera parte se origina desde el CECPAD que aborda de una forma amplia las competencias relacionadas con la Atención a la diversidad que deben tener los profesionales para que haya una verdadera inclusión en el aula por parte del alumnado con algún reto en desarrollo. Se mantienen en el cuestionario las categorías asociadas a las competencias didácticas, organizativas, comunicativas, integradoras y evaluadoras del docente con respecto a la atención a la diversidad y se eliminan las académicas por quedar fuera del alcance del presente estudio.

$Y$, una segunda parte, en las que se han tenido en cuenta algunas cuestiones de la Escala de Evaluación de la Coordinación del CDIAT con la escuela infantil, tales como: la adecuación del intercambio de información entre los profesionales del CDIAT y los docentes; si estos últimos tienen suficiente información sobre el niño y niño atendido por el CDIAT; si el maestro o maestra ha aportado al profesional de atención temprana la información que este le demandaba; si el docente tiene conciencia de que la información del profesional del CDIAT es novedosa con respecto a otros profesionales que trabajan con el menor, e incluso útil para trabajar con las familias o como enriquecimiento personal; y sobe todo si aplican las orientaciones que aporta el profesional de referencia de AT en su trabajo diario en el aula.

Por último, se ha introducido en el cuestionario una pregunta abierta para conocer cómo los docentes mejorarían la relación con el profesional de CDIAT. Pretendiendo obtener conclusiones más específicas sobre la colaboración entre servicios tan importantes para el desarrollo del menor en la etapa de educación infantil. 


\section{Muestra}

Un total de 68 profesores de Educación Infantil de España han participado en el estudio. La muestra es un $97,05 \%$ femenina, con una experiencia profesional diversa sobre todo en centros educativos públicos $(70,58 \%)$.

La recogida de datos se realizó durante el segundo trimestre del 2021. Los objetivos del estudio fueron explicados a los participantes y se indicó el carácter voluntario y confidencial de su participación.

Tabla 1. Caracterización de la muestra

\begin{tabular}{lcc}
\hline Variables descriptivas & Frecuencia & $\%$ \\
\hline Sexo & 2 & \\
Hombre & 66 & 2,94 \\
Mujer & & 97,05 \\
\hline Tramo de edad & 16 & 23,52 \\
$20-25$ años & 17 & 25 \\
$26-35$ años & 25 & 36,76 \\
$36-45$ años & 10 & 14,7 \\
$>45$ años & & \\
Experiencia docente & 11 & 16,17 \\
Ninguna & 15 & 22,05 \\
1-5 años & 11 & 16,17 \\
6-10 años & 24 & 35,29 \\
$11-20$ años & 7 & 10,29 \\
$>20$ años & & \\
Tipo de centro & 48 & 70,58 \\
Público & 15 & 22,05 \\
Concertado & 5 & 7,35 \\
Privado & & \\
\hline
\end{tabular}

\section{Análisis de datos}

El tratamiento de los datos cuantitativos se llevó a cabo a través de SPSS 25.0 donde se realizó un estudio de parámetros descriptivos característicos. Para el análisis de los datos cualitativos se siguieron las fases de reducción, disposición y transformación de los datos, marcadas por Sánchez-Gómez et al. (2017) y se utilizó el software MAXQDA versión 12.

\section{Resultados}

Se presentan los resultados del cuestionario aplicado, presentando la frecuencia y el porcentaje que supone en cada caso, cada nivel de respuesta del ítem, su media y su desviación típica.

\section{Competencias didácticas}

De los datos obtenidos en el bloque de competencias didácticas destaca por su menor puntuación el ítem en el que se solicita al profesorado que valore su capacidad de realizar ajustes y/o adaptaciones necesarias al servicio de las necesidades particulares del alumnado $M=3,84(0,89)$, en el que sólo un $51,47 \%$ se siente moderadamente o muy capacitado. En los ítems sobre la elaboración de la programación del aula, todos presentan porcentajes altos, en el nivel de respuesta de "bastante" capacitados para modificar los puntos de la programación al alumnado con necesidades educativas especiales. 
Por otro lado, cuando se les pregunta si pueden favorecer la adquisición de habilidades para aumentar la autodeterminación, independencia, autonomía y participación del alumnado en su entorno, un $54,41 \%$ de los encuestados indican que favorecen "bastante" la adquisición de estas habilidades, tan importantes en las prácticas centradas en la familia, como es partir del desarrollo funcional del menor.

Por último, hay que destacar que un $47,05 \%$ del profesorado, prioriza los aprendizajes prácticos y funcionales en su aula con todos los alumnos/as y, en especial, con aquellos que presentan algún tipo de necesidad de apoyo especial.

Tabla 2. Nivel de respuesta a los ítems de competencias didácticas

\begin{tabular}{|c|c|c|c|c|c|c|c|c|}
\hline \multirow{2}{*}{ Ítem } & \multicolumn{8}{|c|}{ Nivel de respuesta } \\
\hline & & 1 & 2 & 3 & 4 & 5 & $M$ & SD \\
\hline \multirow{2}{*}{$\begin{array}{l}\text { 5. Soy capaz de realizar los ajustes y/o adaptaciones } \\
\text { necesarias al servicio de las necesidades particulares de cada } \\
\text { alumno/a. }\end{array}$} & $\mathrm{N}$ & 0 & 5 & 18 & 28 & 17 & \multirow{2}{*}{3,84} & \multirow{2}{*}{0,89} \\
\hline & $\%$ & 0 & 7,35 & 26,47 & 41,17 & 25 & & \\
\hline \multirow{2}{*}{$\begin{array}{l}\text { 6. Sé elaborar la programación del aula según los diferentes } \\
\text { ritmos de aprendizaje, capacidades e intereses de mis } \\
\text { alumnos/as. }\end{array}$} & $\mathrm{N}$ & 0 & 5 & 12 & 30 & 21 & \multirow{2}{*}{3,99} & \multirow{2}{*}{0,89} \\
\hline & $\%$ & 0 & 7,35 & 17,64 & 44,11 & 30,88 & & \\
\hline \multirow{2}{*}{$\begin{array}{l}\text { 7. Puedo adaptar los contenidos curriculares para atender a la } \\
\text { diversidad del alumnado. }\end{array}$} & $\mathrm{N}$ & 0 & 3 & 14 & 34 & 17 & \multirow{2}{*}{3,96} & \multirow{2}{*}{0,8} \\
\hline & $\%$ & 0 & 4,41 & 20,58 & 50 & 25 & & \\
\hline \multirow{2}{*}{$\begin{array}{l}\text { 8. Soy capaz de adaptar los objetivos a las diferencias } \\
\text { presentes en el alumnado. }\end{array}$} & $\mathrm{N}$ & 0 & 4 & 12 & 32 & 20 & \multirow{2}{*}{4} & \multirow{2}{*}{0,85} \\
\hline & $\%$ & 0 & 5,88 & 17,64 & 47,05 & 29,41 & & \\
\hline \multirow{2}{*}{$\begin{array}{l}\text { 9. Planteo diferentes actividades en función de los distintos } \\
\text { estilos de aprendizaje y características de mis alumnos y } \\
\text { alumnas. }\end{array}$} & $\mathrm{N}$ & 0 & 3 & 7 & 36 & 22 & \multirow{2}{*}{4,13} & \multirow[t]{2}{*}{0,77} \\
\hline & $\%$ & 0 & 4,41 & 10,29 & 52,94 & 32,35 & & \\
\hline \multirow{2}{*}{$\begin{array}{l}\text { 10. Soy capaz de utilizar recursos diversos para atender a el } \\
\text { alumnado que necesite una atención especial. }\end{array}$} & $\mathrm{N}$ & 0 & 1 & 12 & 32 & 23 & \multirow{2}{*}{4,13} & \multirow{2}{*}{0,75} \\
\hline & $\%$ & 0 & 1,47 & 17,64 & 47,05 & 33,82 & & \\
\hline \multirow{2}{*}{$\begin{array}{l}\text { 11. Utilizo un vocabulario adecuado para dar instrucciones y } \\
\text { compruebo que el niño o niña las ha comprendido. }\end{array}$} & $\mathrm{N}$ & 0 & 0 & 9 & 20 & 39 & \multirow{2}{*}{4,44} & \multirow{2}{*}{0,72} \\
\hline & $\%$ & 0 & 0 & 13,23 & 29,41 & 57,35 & & \\
\hline \multirow{2}{*}{$\begin{array}{l}\text { 12. Soy capaz de desarrollar diversas metodologías para } \\
\text { atender las características individuales de cada alumno/a y } \\
\text { para desarrollar una escuela inclusiva atenta a las diferencias. }\end{array}$} & $\mathrm{N}$ & 0 & 1 & 15 & 29 & 23 & \multirow{2}{*}{4,09} & \multirow{2}{*}{0,79} \\
\hline & $\%$ & 0 & 1,47 & 22,05 & 42,64 & 33,82 & & \\
\hline \multirow{2}{*}{$\begin{array}{l}\text { 13. Propicio una clase activa y participativa para todos mis } \\
\text { alumnos/as. }\end{array}$} & $\mathrm{N}$ & 0 & 0 & 7 & 24 & 37 & \multirow{2}{*}{4,44} & \multirow{2}{*}{0,68} \\
\hline & $\%$ & 0 & 0 & 10,29 & 35,29 & 54,41 & & \\
\hline \multirow{2}{*}{$\begin{array}{l}\text { 14. Promuevo el aprendizaje colaborativo, poniendo al niño o } \\
\text { niña que lo necesita con un compañero/a que le pueda ayudar } \\
\text { a centrar su atención y hacer sus trabajos en clase. }\end{array}$} & $\mathrm{N}$ & 0 & 2 & 12 & 30 & 24 & \multirow{2}{*}{4,12} & 08 \\
\hline & $\%$ & 0 & 2,94 & 17,64 & 44,11 & 35,29 & & 0,0 \\
\hline $\begin{array}{l}\text { 15. Puedo favorecer la adquisición de habilidades para } \\
\text { aumentar la autodeterminación, independencia, autonomía y }\end{array}$ & $\mathrm{N}$ & 0 & 1 & 9 & 37 & 21 & 4,15 & 0,7 \\
\hline participación del alumnado en su entorno. & $\%$ & 0 & 1,47 & 13,23 & 54,41 & 30,88 & & \\
\hline 16. Soy capaz de planificar las clases estableciendo metas & $\mathrm{N}$ & 0 & 1 & 13 & 32 & 22 & & 076 \\
\hline cuenta los aprendizajes de su nivel. & $\%$ & 0 & 1,47 & 19,11 & 47,05 & 32,35 & & 0,10 \\
\hline $\begin{array}{l}\text { 17. Priorizo los aprendizajes prácticos y funcionales en mi aula } \\
\text { con todos los alumnos/as y en especial con aquellos que }\end{array}$ & $\mathrm{N}$ & 0 & 0 & 7 & 29 & 32 & 4,37 & 0,67 \\
\hline presentan algún tipo de atención especial. & $\%$ & 0 & 0 & 10,29 & 42,64 & 47,05 & & \\
\hline
\end{tabular}

\section{Competencias organizativas}

En el bloque de competencias organizativas en atención a la diversidad se puede ver que, en líneas generales, los resultados obtenidos siguen por encima del grado de adquisición del 3,80, tanto en la organización espacial de aula o centro, agrupamientos flexibilizados, revisión de los recursos materiales y didácticos respondiendo a las necesidades de todo el alumnado, como en la temporalización flexible de las rutinas y actividades que se van a trabajar en función de las capacidades de los menores. En la tabla 3 se presentan los resultados obtenidos. 
Tabla 3. Nivel de respuesta a los ítems de competencias organizativas

\begin{tabular}{|c|c|c|c|c|c|c|c|c|}
\hline \multirow[b]{2}{*}{ Ítem } & \multicolumn{8}{|c|}{ Nivel de respuesta } \\
\hline & & 1 & 2 & 3 & 4 & 5 & $\mathrm{M}$ & SD \\
\hline \multirow{2}{*}{$\begin{array}{l}\text { 18. Puedo organizar el espacio en el aula, favoreciendo la } \\
\text { atención a la diversidad del alumnado. }\end{array}$} & $\mathrm{N}$ & 0 & 1 & 6 & 30 & 31 & \multirow{2}{*}{4,34} & \multirow{2}{*}{0,7} \\
\hline & $\%$ & 0 & 1,47 & 8,82 & 44,11 & 45,58 & & \\
\hline \multirow{2}{*}{$\begin{array}{l}\text { 19. Sé cómo agrupar a los alumnos/as que requieren una } \\
\text { atención especial, dándoles la oportunidad de trabajar y } \\
\text { participar activamente. }\end{array}$} & $\mathrm{N}$ & 0 & 0 & 15 & 29 & 24 & \multirow{2}{*}{4,13} & \multirow{2}{*}{0,75} \\
\hline & $\%$ & 0 & 0 & 22,05 & 42,64 & 35,29 & & \\
\hline \multirow{2}{*}{$\begin{array}{l}\text { 20. Reviso el uso de los recursos materiales o didácticos } \\
\text { regularmente, para que puedan utilizarse de forma flexible y } \\
\text { responder a las necesidades cambiantes de todo el alumnado. }\end{array}$} & $\mathrm{N}$ & 0 & 2 & 13 & 29 & 24 & \multirow{2}{*}{4,1} & \multirow{2}{*}{0,81} \\
\hline & $\%$ & 0 & 2,94 & 19,11 & 42,64 & 35,29 & & \\
\hline \multirow{2}{*}{$\begin{array}{l}\text { 21. Unifico estrategias educativas con todos los demás } \\
\text { profesionales que inciden en el grupo-clase. }\end{array}$} & $\mathrm{N}$ & 1 & 6 & 15 & 23 & 23 & \multirow{2}{*}{3,89} & \multirow{2}{*}{1,01} \\
\hline & $\%$ & 1,47 & 8,82 & 22,05 & 33,82 & 33,82 & & \\
\hline \multirow{2}{*}{$\begin{array}{l}\text { 22. Utilizo los tiempos, de forma flexible, en función de la } \\
\text { actividad propuesta y las necesidades del alumnado. }\end{array}$} & $\mathrm{N}$ & 0 & 1 & 10 & 33 & 24 & \multirow{2}{*}{4,1} & \multirow{2}{*}{0,81} \\
\hline & $\%$ & 0 & 1,47 & 14,7 & 48,52 & 35,29 & & \\
\hline \multirow{2}{*}{$\begin{array}{l}\text { 23. Organizo el espacio de aula de acuerdo con la planificación } \\
\text { y objetivos de aprendizaje planteados. }\end{array}$} & $\mathrm{N}$ & 0 & 2 & 7 & 28 & 31 & \multirow{2}{*}{4,29} & \multirow{2}{*}{0,76} \\
\hline & $\%$ & 0 & 2,94 & 10,29 & 41,17 & 45,58 & & \\
\hline \multirow{2}{*}{$\begin{array}{l}\text { 24. Utilizo modalidades flexibles de agrupación cuando trabajo } \\
\text { con alumnos/as que requieren algún tipo de atención especial. }\end{array}$} & $\mathrm{N}$ & 0 & 3 & 9 & 33 & 23 & \multirow{2}{*}{4,1} & \multirow{2}{*}{0,81} \\
\hline & $\%$ & 0 & 4,41 & 13,23 & 48,52 & 33,82 & & \\
\hline \multirow{2}{*}{$\begin{array}{l}\text { 25. Organizo el aula con distintos espacios fijos (rincones, } \\
\text { biblioteca, etc.). }\end{array}$} & $\mathrm{N}$ & 2 & 4 & 5 & 17 & 40 & \multirow{2}{*}{4,3} & \multirow{2}{*}{1,03} \\
\hline & $\%$ & 2,94 & 5,88 & 7,35 & 25 & 58,82 & & \\
\hline \multirow{2}{*}{$\begin{array}{l}\text { 26. Utilizo otros espacios distintos al aula para realizar las } \\
\text { actividades (patio, biblioteca, gimnasio, etc.). }\end{array}$} & $\mathrm{N}$ & 2 & 2 & 6 & 17 & 41 & \multirow{2}{*}{4,36} & \multirow{2}{*}{0,96} \\
\hline & $\%$ & 2,94 & 2,94 & 8,82 & 25 & 60,29 & & \\
\hline \multirow{2}{*}{$\begin{array}{l}\text { 27. He identificado que la escuela cuenta con las condiciones } \\
\text { de accesibilidad para todos los estudiantes. }\end{array}$} & $\mathrm{N}$ & 2 & 5 & 17 & 20 & 24 & \multirow{2}{*}{3,87} & \multirow{2}{*}{1,06} \\
\hline & $\%$ & 2,94 & 7,35 & 25 & 29,41 & 35,29 & & \\
\hline
\end{tabular}

De los datos mostrados llama la atención, el ítem que pregunta sobre la unificación de estrategias educativas con todos los demás profesionales que inciden en el grupo-clase, ya que hay un variado número de respuestas, visualizando que es difícil unificar criterios con otros profesionales, detrás de su práctica docente. Por otro lado, el que haya docentes que todavía no identifiquen $(2,94 \%)$ o hayan identificado pocas condiciones de accesibilidad (7,35\%) para todos los estudiantes en su centro, es motivo de preocupación debido a que la accesibilidad universal es un derecho del menor.

\section{Competencias comunicativas}

En la categoría sobre las competencias comunicativas en atención a la diversidad, predomina los valores altos en el grado de adquisición de esta competencia, con una media de 4,42 de valoración de los ítems. A continuación, en la tabla 4, se pueden observar las respuestas dadas por los encuestados.

Tabla 4. Nivel de respuesta a los ítems de competencias comunicativas

\begin{tabular}{|c|c|c|c|c|c|c|c|c|}
\hline \multirow[b]{2}{*}{ Ítem } & \multicolumn{8}{|c|}{ Nivel de respuesta } \\
\hline & & 1 & 2 & 3 & 4 & 5 & $M$ & SD \\
\hline \multirow{2}{*}{$\begin{array}{l}\text { 28. Soy capaz de comunicarme con mis alumnos/as a través de } \\
\text { distintos códigos, dando respuesta a sus necesidades. }\end{array}$} & $\mathrm{N}$ & 0 & 3 & 14 & 29 & 22 & \multirow{2}{*}{4,02} & \multirow{2}{*}{0,83} \\
\hline & $\%$ & 0 & 4,41 & 20,58 & 42,64 & 32,35 & & \\
\hline \multirow{2}{*}{$\begin{array}{l}\text { 29. Permito que todos mis alumnos/as expresen libremente sus } \\
\text { ideas y emociones, y les escucho. }\end{array}$} & $\mathrm{N}$ & 0 & 0 & 3 & 19 & 46 & \multirow{2}{*}{4,63} & \multirow{2}{*}{0,56} \\
\hline & $\%$ & 0 & 0 & 4,41 & 27,94 & 67,64 & & \\
\hline \multirow[t]{2}{*}{ 30. Soy capaz de gestionar las opiniones y voces de los alumnos. } & $\mathrm{N}$ & 0 & 0 & 10 & 23 & 35 & \multirow{2}{*}{4,36} & \multirow{2}{*}{0,72} \\
\hline & $\%$ & 0 & 0 & 14,70 & 33,82 & 51,47 & & \\
\hline \multirow{2}{*}{$\begin{array}{l}\text { 31. Informo a las familias, así como a los docentes de los siguientes } \\
\text { años, acerca del proceso y los resultados educativos de los niño/as. }\end{array}$} & $\mathrm{N}$ & 0 & 0 & 4 & 17 & 47 & \multirow{2}{*}{4,63} & \multirow{2}{*}{0,59} \\
\hline & $\%$ & 0 & 0 & 5,88 & 25 & 69,11 & & \\
\hline \multirow{2}{*}{$\begin{array}{l}\text { 32. Soy capaz de ser flexible con el profesorado para poner en } \\
\text { marcha los frutos del diálogo, actividades y planteamientos } \\
\text { propuestos. }\end{array}$} & $\mathrm{N}$ & 0 & 0 & 6 & 32 & 30 & \multirow{2}{*}{4,35} & \multirow{2}{*}{0,63} \\
\hline & $\%$ & 0 & 0 & 8,82 & 47,05 & 44,11 & & \\
\hline \multirow{2}{*}{$\begin{array}{l}\text { 33. Soy capaz de trabajar de manera colaborativa con todos los } \\
\text { profesionales de la escuela. }\end{array}$} & $\mathrm{N}$ & 0 & 1 & 8 & 21 & 38 & \multirow{2}{*}{4,41} & \multirow{2}{*}{0,75} \\
\hline & $\%$ & 0 & 1,47 & 11,76 & 30,88 & 55,88 & & \\
\hline \multirow{2}{*}{$\begin{array}{l}\text { 34. Facilito que mi alumnado se exprese a través de diversas } \\
\text { formas y medios de expresión. }\end{array}$} & $\mathrm{N}$ & 0 & 0 & 2 & 25 & 41 & \multirow{2}{*}{4,57} & \multirow{2}{*}{0,55} \\
\hline & $\%$ & 0 & 0 & 2,94 & 36,76 & 60,29 & & \\
\hline
\end{tabular}


Destaca que un $4,41 \%$ del profesorado se perciba con poca capacidad, o moderadamente capacitados $(20,58 \%)$ para comunicarse con su alumnado por medio de distintos códigos para poder dar respuesta a sus necesidades.

Resaltar el $69,11 \%$ indica que se siente muy capacitado para informar a las familias, así como a los docentes de los siguientes años, acerca del proceso y los resultados educativos de los niños y niñas; resaltando en esta respuesta la importancia de que haya una buena comunicación entre profesionales.

Por el contrario, indican que es costoso trabajar de manera colaborativa con los otros docentes del centro escolar, ya que hay más disparidad de opiniones, un $55,88 \%$ se sienten "muy" capacitados, un $30,88 \%$ de las respuestas "bastante"; y un $11,76 \%$ de los encuestados "moderadamente" desarrollan esta competencia de colaboración. Incluso la capacitación de ser flexible con el profesorado para poner en marcha los frutos del diálogo, actividades y planteamientos propuestos, las respuestas oscilan entre, un $44,11 \%$ de los que se sienten muy flexibles para trabajar con este método, un $47,05 \%$ bastante y un $8,82 \%$ moderadamente capacitado.

\section{Competencias integradoras}

Las competencias integradoras en la atención a la diversidad obtienen una media de 4,28 . En donde muchos de sus ítems superan la valoración de "mucho" entre un 33,83 $\%$ y un $63,23 \%$ de las respuestas dadas.

En cambio, cuando se pregunta por si el profesorado utiliza las nuevas tecnologías para favorecer la inclusión de los alumnos/as que necesitan una atención especial se obtiene una media de 3,73.

Tabla 5. Nivel de respuesta a los ítems de competencias integradoras

\begin{tabular}{|c|c|c|c|c|c|c|c|c|}
\hline \multirow[b]{2}{*}{ Ítem } & \multicolumn{8}{|c|}{ Nivel de respuesta } \\
\hline & & 1 & 2 & 3 & 4 & 5 & $\mathrm{M}$ & SD \\
\hline \multirow{2}{*}{$\begin{array}{l}\text { 35. Integro alumnos/as con diferentes niveles y capacidades } \\
\text { en distintos grupos. }\end{array}$} & $\mathrm{N}$ & 3 & 1 & 4 & 28 & 32 & \multirow{2}{*}{4,25} & \multirow{2}{*}{0,96} \\
\hline & $\%$ & 4,41 & 1,47 & 5,88 & 41,17 & 47,05 & & \\
\hline \multirow{2}{*}{$\begin{array}{l}\text { 36. Soy capaz de atender racionalmente la diversidad del aula, } \\
\text { poniendo en práctica el principio de la equidad. }\end{array}$} & $\mathrm{N}$ & 0 & 1 & 8 & 33 & 26 & \multirow{2}{*}{4,23} & \multirow{2}{*}{0,70} \\
\hline & $\%$ & 0 & 1,47 & 11,76 & 48,52 & 38,23 & & \\
\hline \multirow{2}{*}{$\begin{array}{l}\text { 37. Utilizo los conocimientos previos de los alumnos/as para } \\
\text { crear situaciones de aprendizaje relacionadas con los centros } \\
\text { de interés a trabajar en clase. }\end{array}$} & $\mathrm{N}$ & 0 & 0 & 3 & 35 & 30 & \multirow{2}{*}{4,39} & \multirow{2}{*}{0,57} \\
\hline & $\%$ & 0 & 0 & 4,41 & 51,47 & 44,11 & & \\
\hline \multirow{2}{*}{$\begin{array}{l}\text { 38. Soy capaz de desarrollar prácticas incluyentes, de manera } \\
\text { que todos los alumnos/as tengan las mismas oportunidades de } \\
\text { aprender independientemente de sus circunstancias. }\end{array}$} & $\mathrm{N}$ & 0 & 1 & 8 & 29 & 30 & \multirow{2}{*}{4,29} & \multirow{2}{*}{0,72} \\
\hline & $\%$ & 0 & 1,47 & 11,76 & 42,64 & 44,11 & & \\
\hline \multirow{2}{*}{$\begin{array}{l}\text { 39. Transmito a mis alumnos /as la idea de que la convivencia } \\
\text { hay que entenderla como una tarea de todos. }\end{array}$} & $\mathrm{N}$ & 0 & 0 & 5 & 20 & 43 & \multirow{2}{*}{4,55} & \multirow{2}{*}{0,62} \\
\hline & $\%$ & 0 & 0 & 7,35 & 29,41 & 63,23 & & \\
\hline \multirow{2}{*}{$\begin{array}{l}\text { 40. Facilito a mis alumnos/as el aprendizaje para la resolución } \\
\text { dialogada de conflictos. }\end{array}$} & $\mathrm{N}$ & 0 & 0 & 4 & 25 & 39 & \multirow{2}{*}{4,51} & \multirow{2}{*}{0,6} \\
\hline & $\%$ & 0 & 0 & 5,88 & 36,76 & 57,35 & & \\
\hline \multirow{2}{*}{$\begin{array}{l}\text { 41. Refuerzo y atiendo a los alumnos/as con algún tipo de } \\
\text { dificultad, así como a los alumnos brillantes. }\end{array}$} & $\mathrm{N}$ & 0 & 0 & 6 & 24 & 38 & \multirow{2}{*}{4,47} & \multirow{2}{*}{0,65} \\
\hline & $\%$ & 0 & 0 & 8,82 & 35,29 & 55,88 & & \\
\hline \multirow{2}{*}{$\begin{array}{l}\text { 42. Utilizo las nuevas tecnologías para favorecer la inclusión } \\
\text { de los alumnos/as que necesitan una atención especial. }\end{array}$} & $\mathrm{N}$ & 5 & 4 & 18 & 18 & 23 & \multirow{2}{*}{3,73} & \multirow{2}{*}{1,19} \\
\hline & $\%$ & 7,35 & 5,88 & 26,47 & 26,47 & 33,82 & & \\
\hline \multirow{2}{*}{$\begin{array}{l}\text { 43. Ofrezco apoyo psicopedagógico al alumnado que lo } \\
\text { necesite. }\end{array}$} & $\mathrm{N}$ & 0 & 4 & 15 & 22 & 27 & \multirow{2}{*}{4,05} & \multirow{2}{*}{0,92} \\
\hline & $\%$ & 0 & 5,88 & 22,05 & 32,35 & 39,7 & & \\
\hline \multirow{2}{*}{$\begin{array}{l}\text { 44. Implico a las familias de todos mis alumnos en el proceso } \\
\text { de enseñanza aprendizaje. }\end{array}$} & $\mathrm{N}$ & 0 & 1 & 10 & 20 & 37 & \multirow{2}{*}{4,36} & \multirow{2}{*}{0,78} \\
\hline & $\%$ & 0 & 1,47 & 14,7 & 29,41 & 54,41 & & \\
\hline
\end{tabular}




\section{Competencias evaluadoras}

En el bloque de competencias evaluadoras en atención a la diversidad se puede ver que, en líneas generales, los resultados obtenidos están por encima del grado de adquisición del 4,3. La mayoría de los sujetos valoran positivamente esta competencia, teniéndola adquirida, resaltando los resultados que se destacan a continuación (tabla $6)$.

Tabla 6. Nivel de respuesta a los ítems de competencias evaluadoras

\begin{tabular}{|c|c|c|c|c|c|c|c|c|}
\hline \multirow[b]{2}{*}{ İtem } & \multicolumn{8}{|c|}{ Nivel de respuesta } \\
\hline & & 1 & 2 & 3 & 4 & 5 & $\mathrm{M}$ & SD \\
\hline \multirow{2}{*}{$\begin{array}{l}\text { 45. Soy capaz de diferenciar cuándo un alumno/a tiene que ser } \\
\text { diagnosticado por el orientador del centro. }\end{array}$} & $\mathrm{N}$ & 0 & 0 & 14 & 29 & 25 & \multirow{2}{*}{4,16} & \multirow{2}{*}{0,73} \\
\hline & $\%$ & 0 & 0 & 20,58 & 42,64 & 36,76 & & \\
\hline \multirow{2}{*}{$\begin{array}{l}\text { 46. Realizo una evaluación inicial para conocer el nivel de } \\
\text { aprendizajes previos de todo el alumnado. }\end{array}$} & $\mathrm{N}$ & 1 & 3 & 10 & 18 & 36 & \multirow{2}{*}{4,25} & \multirow{2}{*}{0,96} \\
\hline & $\%$ & 1,47 & 4,41 & 14,7 & 26,47 & 52,94 & & \\
\hline \multirow{2}{*}{$\begin{array}{l}\text { 47. Soy capaz de hacer una evaluación individualizada en } \\
\text { función del alumnado que tengo. }\end{array}$} & $\mathrm{N}$ & 0 & 1 & 8 & 24 & 35 & \multirow{2}{*}{4,36} & \multirow{2}{*}{0,74} \\
\hline & $\%$ & 0 & 1,47 & 11,76 & 35,29 & 51,47 & & \\
\hline \multirow{2}{*}{$\begin{array}{l}\text { 48. Manejo modelos evaluativos flexibles y diversificados para } \\
\text { evaluar el aprendizaje del alumnado. }\end{array}$} & $\mathrm{N}$ & 0 & 1 & 15 & 29 & 23 & \multirow{2}{*}{4,08} & \multirow{2}{*}{0,78} \\
\hline & $\%$ & 0 & 1,47 & 22,05 & 42,64 & 33,82 & & \\
\hline \multirow{2}{*}{$\begin{array}{l}\text { 49. Recojo información de forma periódica para evaluar los } \\
\text { procesos de aprendizaje de todos mis alumnos/as. }\end{array}$} & $\mathrm{N}$ & 0 & 0 & 10 & 23 & 35 & \multirow{2}{*}{4,36} & \multirow{2}{*}{0,72} \\
\hline & $\%$ & 0 & 0 & 14,7 & 33,82 & 51,47 & & \\
\hline \multirow{2}{*}{$\begin{array}{l}\text { 50. Utilizo instrumentos variados de evaluación, según la } \\
\text { actividad y la competencia a evaluar. }\end{array}$} & $\mathrm{N}$ & 0 & 0 & 13 & 28 & 27 & \multirow{2}{*}{4,2} & \multirow{2}{*}{0,73} \\
\hline & $\%$ & 0 & 0 & 19,11 & 41,17 & 39,7 & & \\
\hline \multirow{2}{*}{$\begin{array}{l}\text { 51. Realizo una evaluación de todos los alumnos/as al final del } \\
\text { proceso educativo. }\end{array}$} & $\mathrm{N}$ & 0 & 0 & 7 & 19 & 42 & \multirow{2}{*}{4,51} & \multirow{2}{*}{0,67} \\
\hline & $\%$ & 0 & 0 & 10,29 & 27,94 & 61,76 & & \\
\hline \multirow{2}{*}{$\begin{array}{l}\text { 52. Evalúo los procesos de enseñanza y el desarrollo de mi } \\
\text { práctica docente ejercida con todo el alumnado. }\end{array}$} & $\mathrm{N}$ & 0 & 2 & 10 & 31 & 25 & \multirow{2}{*}{4,16} & \multirow{2}{*}{0,77} \\
\hline & $\%$ & 0 & 2,94 & 14,7 & 45,58 & 36,76 & & \\
\hline \multirow{2}{*}{$\begin{array}{l}\text { 53. Priorizo los aprendizajes prácticos y funcionales en mi aula } \\
\text { con todos los alumnos/as y en especial con aquellos que } \\
\text { presentan necesidades educativas especiales. }\end{array}$} & $\mathrm{N}$ & 0 & 0 & 8 & 24 & 36 & \multirow[b]{2}{*}{4,41} & \multirow{2}{*}{0,69} \\
\hline & $\%$ & 0 & 0 & 11,76 & 35,29 & 52,94 & & \\
\hline \multirow{2}{*}{$\begin{array}{l}\text { 54. Soy éticamente correcto/a a la hora de evaluar a todo el } \\
\text { alumnado. }\end{array}$} & $\mathrm{N}$ & 0 & 0 & 4 & 24 & 40 & \multirow{2}{*}{4,52} & \multirow{2}{*}{0,6} \\
\hline & $\%$ & 0 & 0 & 5,88 & 35,29 & 58,82 & & \\
\hline
\end{tabular}

Destaca un alto porcentaje en la escala con la puntuación en los procesos de evaluación inicial para conocer el nivel de aprendizajes previos de todo el alumnado (52,94\% de los encuestados), evaluación individualizada en función del alumnado que se tenga ( $51,47 \%$ de la muestra); y evaluación de todos los alumnos y alumnas al final del proceso educativo $(61,76 \%$ de las contestaciones al ítem).

Con respecto, a si el profesorado utiliza instrumentos variados de evaluación, según la actividad y la competencia a evaluar, un $41,17 \%$ de los encuestados indican que los utilizan "bastante". Considerándose, un $58,82 \%$ de los docentes, éticamente correctos a la hora de evaluar a todo el alumnado.

En cuanto a la capacidad de diferenciar cuándo un alumno y alumna tiene que ser diagnosticado por el orientador del centro, un 42,64\% de los encuestados se consideran "bastante" competentes con este ítem.

\section{Coordinación con los profesionales de Atención Temprana}

En último lugar, se presentan los resultados sobre la percepción que el profesorado sobre la coordinación con los profesionales de Atención Temprana. Los resultados obtenidos tienen una media de grado de adquisición del 3,48.

Como se puede ver en la tabla 7 , el $29,41 \%$ de los encuestados consideran "moderado" el intercambio de información entre profesionales del CDIAT con ellos. En cuanto a 
considerar si tienen suficiente información del niño o niña atendido en el centro de desarrollo infantil y atención temprana, el 27,94\% entienden como "moderadamente" o "bastante" esa información que viene por parte de los profesionales del CDIAT. Siendo tan sólo, un $11,76 \%$ los que consideran "mucha" la información proporcionada por el profesional de AT, siendo muy novedosa, diferente a lo que recibe de otros profesionales. Por el contrario, un $41,17 \%$ de la muestra, creen que es "mucha" la información que como docentes ellos ofrecen a los profesionales de AT.

Tras todo este intercambio de información, los docentes indican $30,88 \%$ que "bastantes" aplican las orientaciones que el profesional del CDIAT les ha ofrecido en su trabajo diario. A continuación, se resumen los datos obtenidos en relación con la coordinación interprofesionales (ver tabla 7).

Tabla 7. Nivel de respuesta a los ítems de coordinación entre profesionales

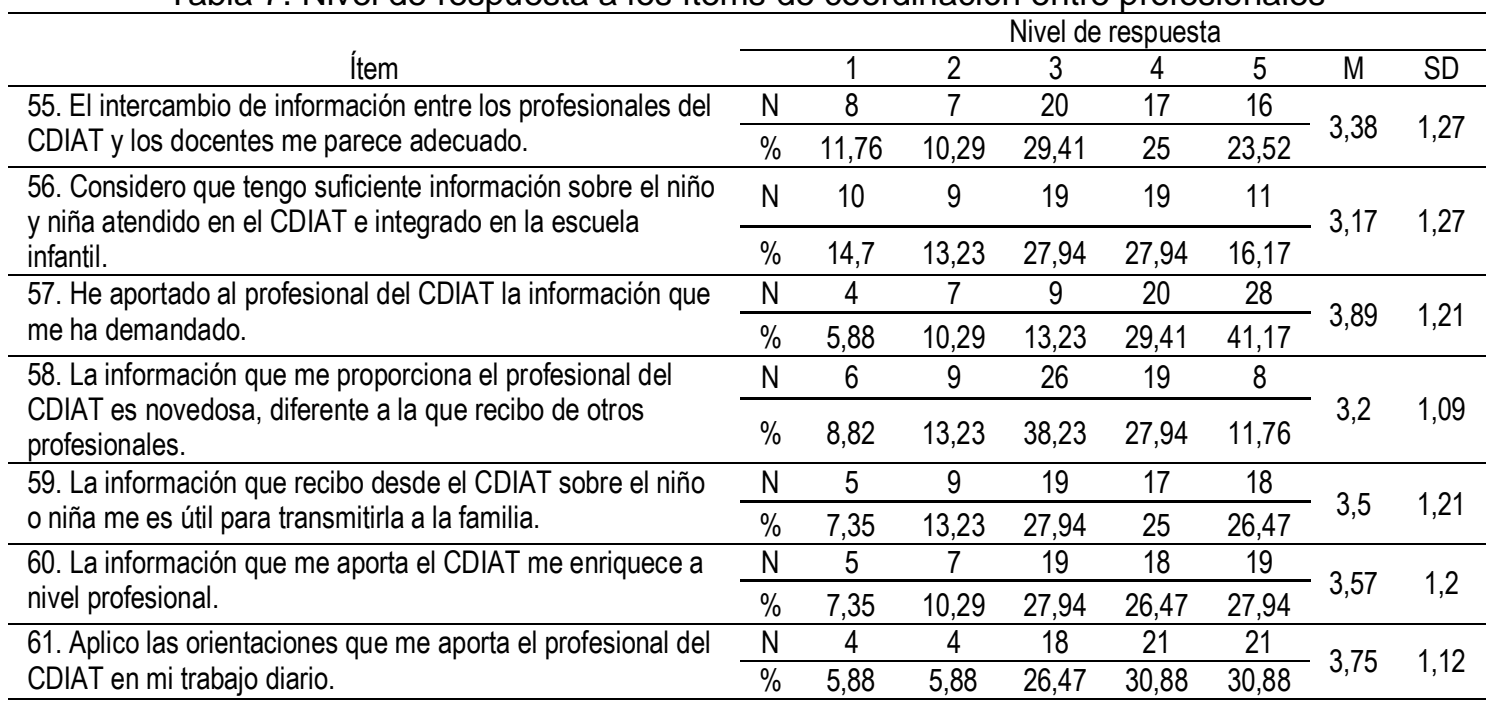

Con respecto al último ítem, cuando se pregunta a los docentes como mejorarían la relación con el equipo de profesionales de CDIAT, tras la realización de un análisis cualitativo de sus respuestas, emergen seis categorías centradas en: las visitas, la comunicación, la información, la coordinación, el contacto y la relación entre profesionales (ver figura 1).

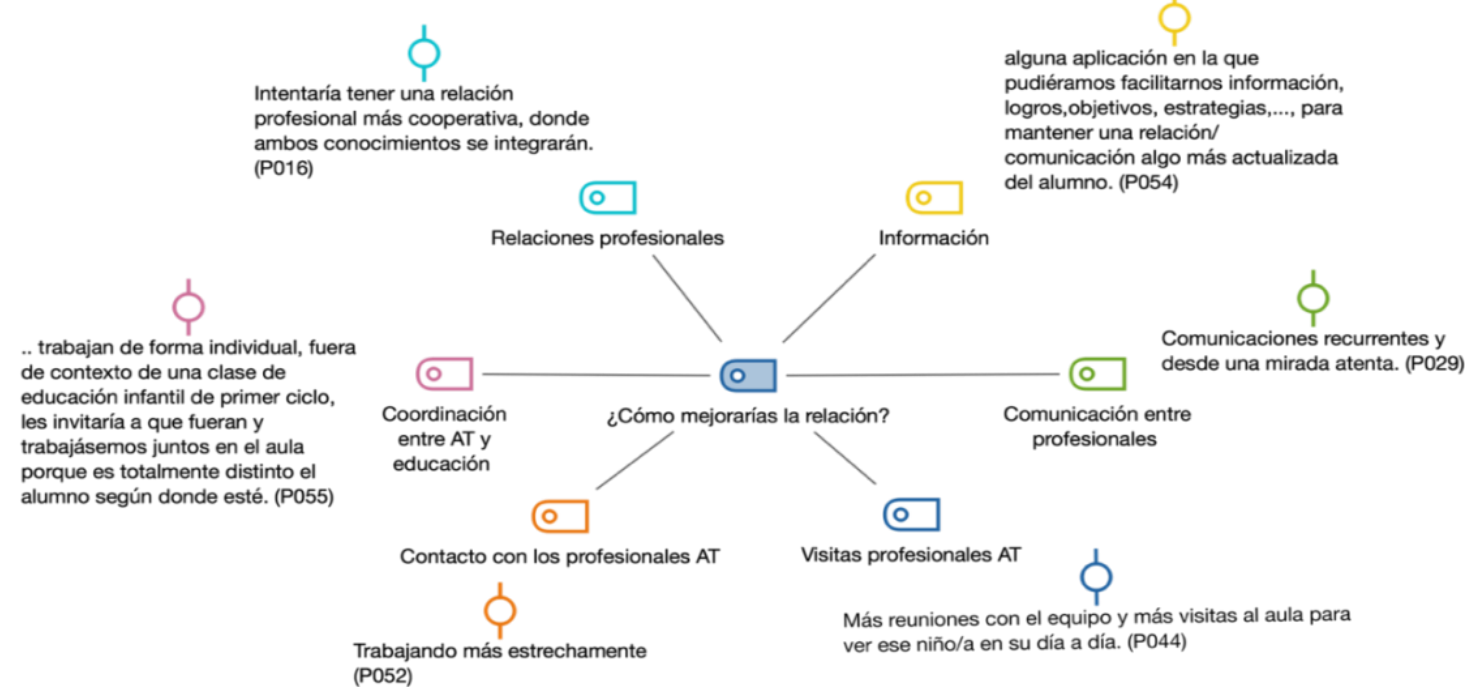

Figura 1. Mapa de códigos con extractos opiniones de los participantes en el estudio 
En primer lugar, con un 52,94\% de respuestas, el profesorado destaca que serían necesario un mayor número de vistas a las aulas, en las que los profesionales de Atención Temprana tuvieran tiempo de observar al menor en el entorno y plantear estrategias conjuntas. Hay que señalar también, que el profesorado es consciente de la carga de trabajo que tienen los CDIAT y que se ve reflejado en las aportaciones realizadas, con un $4,4 \%$ de los encuestados que lo resalta.

En segundo lugar, valoran que debería mejorar la relación entre profesionales del área de educación y AT, conociendo así la forma de trabajo en el aula con el niño o niña, la evolución de estos, las novedades, los avances, etc. Como resalta una docente en su respuesta: "Ellos son los profesionales, pero nosotros conocemos mejor a los niños y cada niño es distinto, aunque tengan el mismo problema. En ocasiones sería aconsejable que tuvieran más en cuenta nuestras valoraciones" (P066).

Por otro lado, un 4,4\% de las respuestas destaca, mejorar la organización y el intercambio de información entre la escuela infantil y el CDIAT, para que la información sea más práctica para el docente sobre actuaciones con el alumnado en concreto. Señalando, que en algunas ocasiones son las propias familias las que facilitan la información de su propio hijo o hija, e incluso las mediadoras entre los profesionales de la escuela y AT. Se destaca la respuesta de una maestra, donde destaca la tardía en el intercambio de información: "Creo que la información del CDIAT, debería llegar al profesorado de manera previa a la escolarización del alumno/a. En la mayoría de los casos llega a la vez o tarde. Esta información es la única herramienta que se tiene en un primer momento, y tanto para las programaciones como para enfrentarnos a la realidad del nuevo/a alumno/a, considero que es de vital importancia para su adaptación y posterior trabajo en el aula y el Centro" (P041).

En tercer lugar, un 4,4\% de las respuestas dadas por los encuestados destacan la buena relación con los centros de desarrollo infantil y atención temprana, proponiendo incluso ideas, tales como: libro viajero con informes escritos de todos los profesionales implicados, para poder acceder a la información más técnica; o alguna aplicación en la que se pudiera facilitar información, logros, objetivos, estrategias, etc. para mantener una relación comunicativa algo más actualizada del alumnado.

Por último, como resalta un $1,57 \%$ de las respuestas, se está abriendo una mirada hacia un modelo más integrador para los menores que presentan necesidades educativas especiales. Actualmente, muchos centros están evolucionando en metodologías alternativas y en ocasiones encontramos con que el modelo metodológico del centro colisiona con los estilos de aprendizaje de los menores. Por ello, es importante que los profesionales de atención temprana conozcan el modelo del centro y busquen estrategias para mejorar la relación entre ambas áreas, ya que chocan enfoques más clínicos con el nuevo modelo centrado en la familia y en los entornos naturales, como es la escuela.

Como se observa en la nube de palabras que hay a continuación (figura 2), destaca la palabra "información", tanto por parte de los profesionales de atención temprana como de la escuela infantil. Siendo la herramienta fundamental para que haya una correcta intervención con el menor, la relación entre profesionales sea fluida y las reuniones periódicas. De esta manera, se formará un equipo entre ambas partes, junto la colaboración principal de las familias, para que haya un programa de coordinación adecuado entre todas las partes. De esta manera, la información que les llegue a los docentes no sólo será por las familias ("en ocasiones sólo nos comunicamos con la familia" p010), a través de reuniones con los profesionales de AT, o cuando les presentan informes elaborados por los equipos de atención temprana. Por ello, en esta 
nube de palabras la relación periódica con la escuela, el contacto estrecho con los docentes, la mirada profesional y teórica con estrategias que ayudarán al educador y la comunicación continua, son un resumen perfecto de lo que los docentes piden para que haya una mejor relación entre ambas áreas.

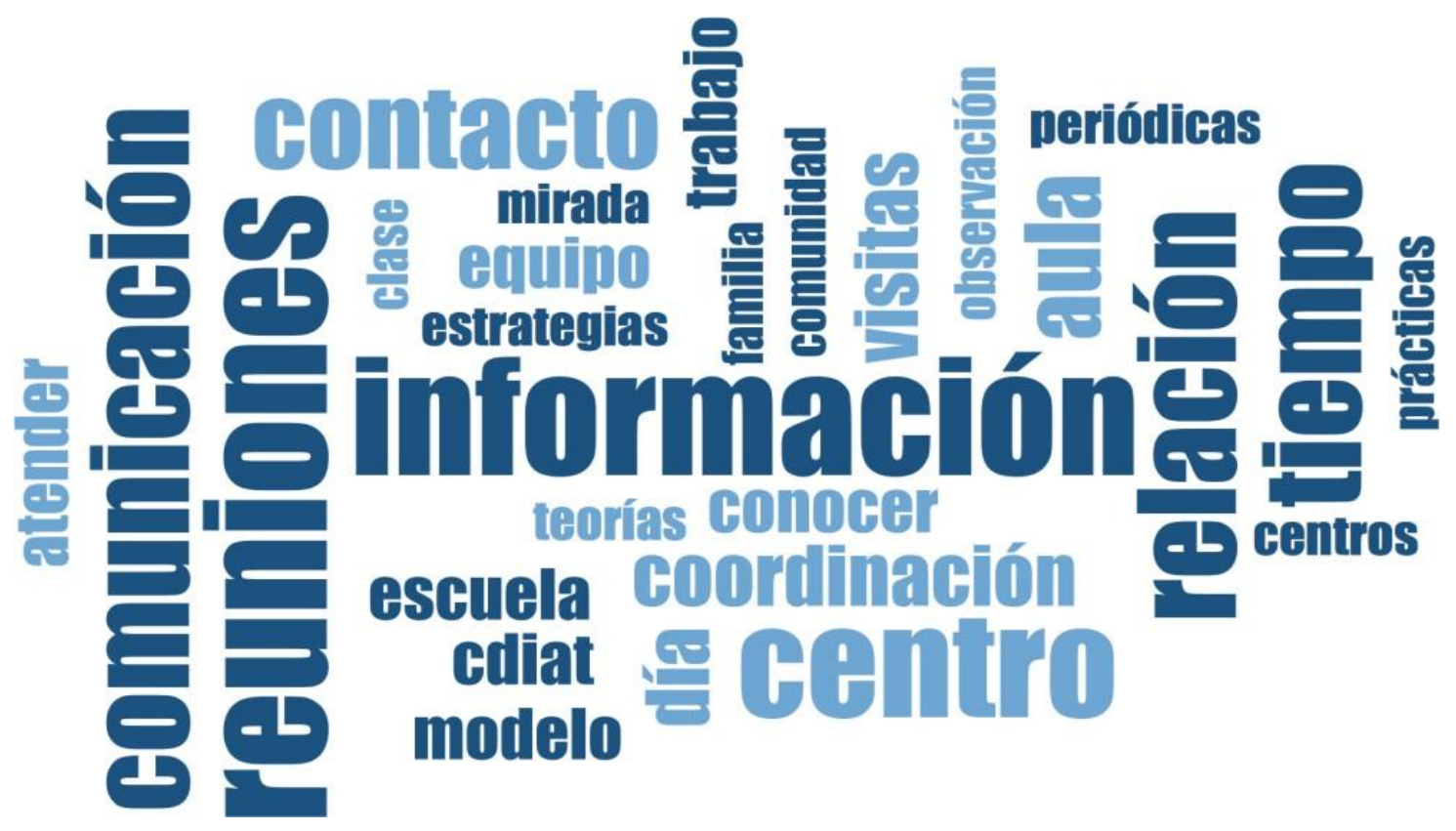

Figura 2. Nube de palabras extraídas de análisis cualitativo

\section{Conclusiones}

Tras el estudio realizado se puede observar en primer lugar la labor tan importante y fundamental que tienen los docentes en su aula cuando en ella hay un alumno o alumna con algún trastorno o riesgo de padecerlo, para reconducir esa atención no sólo a sus necesidades, sino capacitarlos y que su desarrollo funcional vaya poco a poco progresando a su ritmo personal, pero de manera inclusiva en el aula y con el apoyo de todas las personas de referencia que lo rodean: profesorado, compañeros de clase, equipo de orientación educativa, profesionales de Atención Temprana y familia (Grande y González, 2015).

Como se ha podido ver en los resultados aportados las competencias docentes vinculadas con la atención a la diversidad presentan un grado de consecución elevado, tanto a nivel didáctico, organizativo, evaluativo, comunicativo, integrador. Resaltando que las valoraciones más bajas son en su mayoría contestadas por docentes nóveles, con apenas experiencia educativa en el aula y, mucha menos, con casos concretos de atención a la diversidad. Estas cualidades hacen que el trabajo docente esté lleno de personas con un don y un valor especial hacia lo que hacen y, sobre todo, desarrollando en el niño o niña y en sus familias un empoderamiento para estimular y desarrollar en ellos capacidades que antes no tenían o creían que no podían tener. Para ello, los docentes ejercen unas competencias a nivel didáctico, adaptando la programación a los diferentes ritmos de aprendizaje, capacidades e intereses del alumnado; a nivel organizativo, dando oportunidades a todo los alumnas y alumnos con diferentes agrupamientos para que entre ellos se ayuden, o utilizando materiales adaptados, e incluso estructurando el aula para que los objetivos se puedan alcanzar; a nivel comunicativo, en mayor parte utilizando distintos códigos que den respuesta a la necesidad del menor, informando a las familias y docentes de los siguientes años sobre la evolución del alumnado, o trabajando de manera colaborativa con otros profesionales 
que tengan relación con el niño o niña; a nivel integrador, atendiendo la diversidad del aula desde el principio de la equidad, dando las mismas oportunidades de aprendizaje a todos sus alumnos independientemente de sus circunstancias (Martínez y Páramo, 2015); a nivel evaluador, sintiéndose capacitados de detectar signos de alarma con evaluaciones iniciales, continuas y personalizadas para ayudar a ese niño o niña a que lo diagnostiquen correctamente; y por último, a nivel de coordinación con los profesionales de los CDIAT, en donde el nivel de intercambio de información necesita ser mejorado por ambas partes, incluyendo a la familia, pero, sobre todo del profesional AT hacia los docentes donde la información del niño o niña con el que se trabaja debe ser novedosa y útil tanto para el profesorado como para los familiares (García et al., 2012; 2014)

Si nos centramos en el análisis de percepción que tienen el profesorado sobre su relación y colaboración con el equipo de profesionales de los centros de desarrollo infantil y atención temprana (CDIAT), se puede concluir indicando que hay que involucrar más a los profesionales de atención temprana en el centro educativo, es necesario que acudan al aula con más frecuencia, apoyen al docente dentro de la clase y les muestren más estrategias para trabajar con todo el alumnado. Con los resultados se confirma que el profesorado está preparado para atender la diversidad de su alumnado, pero necesitan de ayuda externa para que la atención sea la adecuada, con una coordinación correcta con los profesionales del centro y los de atención temprana, además de la actuación conjunta de la familia, a la que hay que involucrar más en el aula y en la atención asistencial del menor.

Sobre la percepción del profesorado respecto al intercambio de información como la base en la relación docente y profesional de atención temprana se puede indicar que la relación entre los profesionales de CDIAT y los docentes en el aula tiene que mejorar, ya que según el profesorado las visitas al centro escolar no son frecuentes, lo que hace que no conozcan al niño o niña en su entorno natural escolar. El intercambio de información entre los profesionales de ambas áreas, educativa y atención temprana, no es periódica, ni productiva para el trabajo del docente con el alumnado en concreto, lo que no favorece a conocer ni mejorar la intervención del menor. Siendo el docente el cuidador principal, en el horario escolar y quien conocer mejor al menor, por lo que sería aconsejable tener más en cuenta la valoración del profesorado ante el seguimiento del alumno o alumna con signos de alarma. Hay que destacar la importancia del momento adecuado para el trasvase de información entre profesionales, ya que los docentes comentan que no se hace en el momento adecuado. En sus respuestas especifican la importancia de adelantar la reunión y el intercambio de información antes de la escolarización del niño o niña para enfrentarse a la realidad del nuevo alumno o alumna, considerando que es de vital importancia para su adaptación y posterior trabajo en el aula y el centro. Pero lo que más llama la atención es que las familias no forman parte de este proceso de relación entre escuela y CDIAT, siendo la base fundamental del nuevo modelo centrado en la familia.

Cuando se les ha solicitado identificar que aspectos que mejorarían en su relación con el equipo de profesionales de CDIAT, los profesores señalan que la falta de comunicación entre los profesionales de ambas áreas se da con demasiada frecuencia, por lo que este sería el principal punto para mejorar. Otra de las cuestiones, es el poco tiempo que estos profesionales se dedican a trabajar en equipo, debido a la falta de tiempo, al poco personal de AT que puede acudir a los centros o porque su coordinación no es productiva. Esto sería otra cuestión para mejorar que ayudaría a formalizar las actuaciones entre docentes y profesionales de AT, con una coordinación más efectiva entre ambas partes para ayudar el desarrollo de los menores. También se debería de mejorar el tipo de relación, ya que es primordial tener una relación profesional más cooperativa y estrecha, donde ambos conocimientos se integren y complementen para 
plantear estrategias conjuntas. Todo ello está acorde con investigaciones previas sobre el tema (García et al., 2014; Tapia-Gutiérrez et al., 2017; Sheppard y Moran, 2021).

Para concluir, el papel del docente de Educación Infantil es fundamental para conocer todas las características de los hitos del desarrollo del menor de 0-6 años, observar si hay alguna señal de alerta y actuar con protocolos de detección temprana, para que cuando se diagnostique algún caso crear un aula inclusiva, con una organización flexible y personalizada a ese alumnado (Simón et al., 2021). Para que el clima escolar sea completo, es necesario que los profesionales de los CDIAT actúen en coordinación con el docente, orientados y complementados por ambos para llevar a cabo las estrategias idóneas con las que estimular el desarrollo del menor (Garcia et al., 2012; Messiou y Ainscow, 2021).

Tras una minuciosa observación de los resultados, haciendo hincapié en los participantes de la encuesta con características similares en: edad y experiencia laboral; se puede sacar como conclusión que son jóvenes, salidos recientemente de la facultad y sin ninguna experiencia en el área educativa, solamente el contacto que hayan podido tener en sus prácticas. Esto lleva a determinar, como lanzaban Cejudo et al. (2016) en sus pasadas investigaciones, que los universitarios tienen una precaria formación en la atención a la diversidad desde el ámbito educativo, o como sostiene Toral (2019) que la preparación del profesorado en materia de atención a la diversidad ha estado fuera del interés de estos y sólo compete a los especialistas, como son: el maestro de Educación Especial, el de Audición y Lenguaje y el de Pedagogía Terapéutica.

Sandall y Schwartz (2015), destacan la importancia de la formación continua, por parte de los docentes y todos los profesionales que rodean al niño y niña en su entorno natural. Lo que lleva a observar que el profesorado, a lo largo de su experiencia laboral, va mejorando en lo que respecta a la atención a la diversidad, adquiriendo y purificando sus formas de actuar ante el alumnado con necesidades educativas especiales. Pero, en el cuestionario, llama la atención varias veces, como maestras con bastantes años de experiencia laboral, valoran con nota baja la capacidad de realizar ajustes y/o adaptaciones necesarias al servicio de las necesidades particulares de cada alumno/a; la no unificación de criterios con otros profesionales, considerando casi nula la reciprocidad entre las dos áreas de trabajo; y valoran negativamente la información que se da por parte de los CDIAT para poder transmitírsela a las familias. Esto lleva a reflexionar sobre esa formación continua, tan importante en cualquier trabajo, y más aún en la educación, cuando con quien se está tratando e interviniendo es con los menores de 6 años. Se debe cuestionar si esa formación es deber del propio docente, de la escuela o influye la relación alejada con el área de atención temprana, que no puede empoderar la labor docente, al no valorarse las dos áreas mutuamente como es debido, desde el respeto, admiración y complementariedad, pero con un mismo objetivo, el desarrollo íntegro del menor.

\section{Referencias}

Agencia Europea para el Desarrollo de la Educación del Alumnado con Necesidades Educativas Especiales (2012). Formación del profesorado para la Educación Inclusiva. Agencia Europea para el Desarrollo de la Educación del Alumnado con Necesidades Educativas Especiales.

Aguaded, EM, de la Rubia, P y González, E (2013). La importancia de la formación del profesorado en competencias interculturales. Profesorado. Revista de Currículum y Formación del Profesorado, 17(1), 339-365.
Aranda, R y de Andrés C (2004). La organización de la atención temprana en la educación infantil. Tendencias pedagógicas 9 , 217246.

Cejudo, J, Díaz, M, Losada, L y Pérez-González, JC (2016). Necesidades de formación de maestros de infantil y primaria en atención a la diversidad. Sociedad Española de Pedagogía, 68(3), 23-39. 
Division for Early Childhood \& The National Association for the Education of Young Children. (2009). Early childhood inclusion: A joint position statement of the Division for Early Childhood and the National Association for the Education of Young Children. https://www.naeyc.org/files/naeyc/file/positions/DEC_NA EYC_EC_updatedKS.pdf

Dunst, CJ, \& Trivette, CM (2009). Capacity-Building FamilySystems Intervention Practices. Journal of Family Social Work, 12(2), 119-143. https://doi.org/10.1080/10522150802713322

Fluckiger, B, Dunn, J, Stinson, M, \& Wheeley, E (2017). Leading age-appropriate pedagogies in the early years of school. Department of Education and Training, Griffith Institute for Educational Research.

Forlin, C, \& Sin, KF (2017). In-service teacher training for inclusion: Best practice models for professional learning. In $\mathrm{G}$ Noblit (Ed.), Oxford research encyclopedia of education. Oxford University Press.

García, FA, Escorcia, C, Sánchez, M, Orcajada, N y Hernández, E (2014). Atención Temprana Centrada en la Familia. Siglo Cero, 45(3), 6-27.

García, FA, Sánchez, MC, Escorcia, CT y Castellanos, P (2012). Valoración de la coordinación entre Atención Temprana y Educación Infantil por Educadores de Escuelas Infantiles. Edetania. Estudios y propuestas socio- educativas, 41, 145-161.

Grande, P y González, MM (2015). La educación inclusiva en la Educación Infantil: propuestas basadas en la evidencia. Universidad Complutense de Madrid.

Grisham-Brown, J Hemmeter, M, \& Pretti-Frontzac, K (2005). Blended Practices for Teaching Young Children in Inclusive Setting. Paul H. Brookes Publishing Company.

Love, HR, \& Horn, E (2021). Definition, context, quality: Current issues in research examining high-quality inclusive education. Topics in Early Childhood Special Education, 40(4), 204216. https://doi.org/10.1177/2F0271121419846342

Martínez, M y Páramo, M (2015). Una mirada a los procesos cognitivos de atención y planificación en el alumnado en educación infantil. Revista Iberoamericana de Evaluación Educativa, 8(1), 2640.

McLeskey, JL, Rosenberg, MS, \& Westling, DL (2017). Inclusion: Effective practices for all students. Pearson.

Mena, R y García, MP (2017). Cuestionario para la evaluación de competencias del profesorado en atención a la diversidad. Universidad de Murcia.

Messiou, K, \& Ainscow, M (2021). Inclusive inquiry: An innovative approach for promoting inclusion in schools. Revista Latinoamericana de Educación Inclusiva, 15(2), 23-37. https://doi.org/10.4067/S0718-73782021000200023
Núñez, L y Duarte, A (2011). Percepciones de los profesionales sobre la coordinación institucional en la atención temprana en Andalucía. Educación y Diversidad, 5 (2), 155-168.

Orcajada, N (2017). Diseño, aplicación y evaluación del plan de formación de tutores de atención temprana. Tesis doctoral, Universidad de Murcia, Murcia, España.

Rodríguez, H (2019). La Formación inicial del profesorado para la inclusión. Un urgente desafío que es necesario atender. Publicaciones: Facultad de Educación y Humanidades del Campus de Melilla, 49(3), 211-225.

Sánchez-Gómez, MC, Martín Cilleros, MV, García Peñalvo, FJ, Muñoz Sánchez, JL, Pinto, A, Parra, E y Franco, M (2017). Análisis de contenido cualitativo: Estudio de la satisfacción de los usuarios sobre la presentación de un nuevo medicamento en la salud pública. En AP Costa, MC Sánchez-Gómez y MV Martín-Cilleros (Eds.), La Práctica de la investigación cualitativa: Ejemplificación de Estudios (pp. 57-92). Ludomedia.

Sandall, S y Schwartz, IS (2015). Apoyar paso a paso. El aprendizaje de niños y niñas con necesidades especiales en el aula de infantil. Ambits de psicopedagogia i orientació, 43, 83-85.

Schilling, C (2018). Formación docente. Experiencias de prácticas pedagógicas y trabajo comunitario como espacio de reconocimiento. En D Ferrada (Ed.), Reflexiones y experiencias educativas desde las comunidades (pp. 149-172). Ediciones UCM.

Sheppard, ME, \& Moran, KK (2021). The Role of Early Care Providers in Early Intervention and Early Childhood Special Education Systems. Early Childhood Education Journal, 1-11. https://doi.org/10.1007/s10643-021-01225-x

Simón, C, Echeita, G, Sandoval, M y Dios, M (2021). Creación de una Estructura Colaborativa entre el Alumnado, Docentes e Investigadores: Impacto en la Mejora Docente. Revista latinoamericana de educación inclusiva, 15(2), 97-110. https://doi.org/10.4067/S0718-73782021000200097

Tapia-Gutierrez, C, Palma-Mardones, A y González-Parra, K (2017). Atención temprana, percepción de madres de niños y niñas con Síndrome de Down. En Revista Actualidades Investigativas en Educación, 17(1), 1-22. https://doi.org/10.15517/aie.v17i1.27226

Toral, A (2019). Detección y atención temprana de las dificultades en el aprendizaje de niños y niñas de segundo grado de la básica elemental. [Trabajo Final de Máster. Universidad Laica Vicente Rocafuerte]. Guayaquil.

UNESCO (2020a). Towards inclusion in education: Status, trends and challenges. The UNESCO Salamanca Statement 25 years on. UNESCO. https://unesdoc.unesco.org/ark:/48223/pf0000375748

UNESCO (2020b). Global Education Monitoring Report, 2020: Inclusion and Education: All Means All. UNESCO. https://gemreport-2020.unesco.org/ 